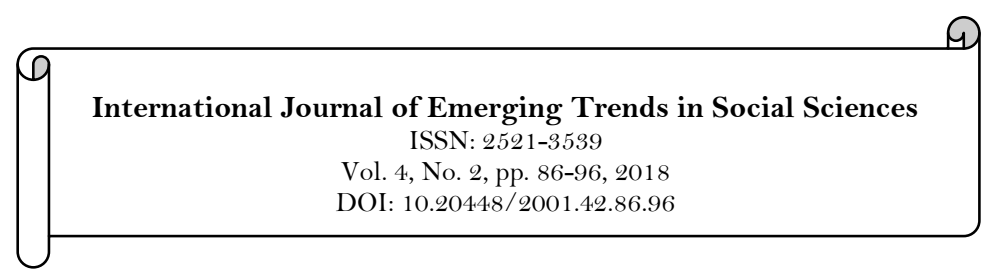

Uupdates

\title{
Antecedents of Citation Impact and Intention to Publish on Open Access Journals: A Case of Agricultural Research Institutes Tanzania
}

\author{
Zainabu Zuberi Massoro's \\ Roslina Othman ${ }^{2}$ \\ ${ }^{1}$ The Mwalimu Nyerere Memorial Academy -Tanzania. \\ Email:zainabuzm@gmail.com \\ ${ }^{2}$ International Islamic University - Malaysia.
}

\begin{tabular}{|c|c|}
\hline Abstract & \\
\hline $\begin{array}{l}\text { This study assessed the factors that contribute to intention to publish in } \\
\text { open access journals among researchers in Tanzanian Agricultural } \\
\text { Research Institutes. Five factors derived from the literature were used to } \\
\text { predict intention to publish in Open Access Journals. These factors are: } \\
\text { journal reputation, the speed of publishing, content relevance, visible } \\
\text { advantage and citation impact. Data were collected from } 121 \text { researchers } \\
\text { through a cross-section survey questionnaire. The findings revealed that } \\
\text { journal reputation, visibility advantage and citation impact have } \\
\text { significant effect on intention to publish in open access journals. } \\
\text { Nonetheless, further analysis showed that the speed of publishing as well } \\
\text { as content relevance have no significant influence on citation impact. } \\
\text { This article articulates the basis for research institutions and } \\
\text { practitioners to nurture open access publishing in order to address the } \\
\text { dearth of empirical studies on open access journal publishing in } \\
\text { Tanzania. }\end{array}$ & $\begin{array}{l}\text { Keywords: } \\
\text { Citation impact } \\
\text { Intention to publish } \\
\text { Open access journals } \\
\text { Publishing } \\
\text { Predictors } \\
\text { Agricultural research institutes } \\
\text { Tanzania. } \\
\text { Licensed: } \\
\text { This work is licensed under a } \\
\text { Creative Commons Attribution } \\
\text { 4.0 License. } \\
\text { Publisher: } \\
\text { Scientific Publishing Institute }\end{array}$ \\
\hline
\end{tabular}

\section{Introduction}

The invention of Information Technology (IT) has contributed significantly to the development of scholarly communication, including electronic publishing commonly known as Open Access Journal (OAJ). The initiation of OAJ resulted from concerted efforts in liberalizing knowledge and information such as Budapest Open Access Initiative (2002) Bethesda statement on open access of 2003 (Brown et al., 2003) and (Berlin Declaration on OA Knowledge, 2003).

Thus, owing to its free availability online, OAJ publishing has become one of the popular media for extensive dissemination of knowledge among researchers, academics and scholars. The OAJ is regarded as a saviour since the barriers associated with publication have been significantly reduced (Joshi, Vatnal, \& Manjunath, 2012) and the pace of research dissemination has improved compared to the traditional model of publishing (Suber, 2004). The availability of free access offered by OAJ is considered as a means of paying back the public funds spent on research to a wider audience (Ware \& Mabe, 2015). Thus, OAJ is a kernel of a knowledge-based society as it provides ubiquitous access.

The initiation of OAJ has necessitated the establishment of the Directory of Open Access Journal (DOAJ) with aims of facilitating registration, indexing and quality assurance (Miguel, Tannuri, \& Grácio, 2016). The DOAJ is regarded as an online community-curate which provides access to high quality open access peer reviewed journals. The presence of DOAJ has motivated the growth of OAJ, where a total of 9,158 peerreviewed open access journals were registered and indexed in 2015 (Masrek \& Yaakub, 2015). Subsequently, the OAJ has received remarkable recognition from international databases such as Web of Science and SCOPUS. 


\section{Literature Review}

The trend on OAJ publishing exhibits tremendous development among the developed countries compared to developing countries. Contrary to expectation, the mining of literature reveals that researchers in Sub Saharan Africa (SSA) are still lagging behind in OAJ publishing. Ware and Mabe (2015) and Nwagwu (2013) documented a negligible contribution made by SSA on publishing in OAJ. The statistics presented in the DOAJ also shows that out of 11,260 registered open-access journals, only 125 originate from SSA (Directory of Open Access Journal, 2016). Francescon (2017) study on research without boarder demonstrates that Africa shares less than one per cent of the world's research output which limits knowledge sharing. Most of the African scientific findings are either published in un-indexed journals or remain unpublished (Francescon, 2017; Ware \& Mabe, 2015) which limits collaboration among researchers. Consequently, the existence of negligible contribution in the OAJ publishing limits article citations and visibility of research products. The presence of minimal number of articles in OAJ could be associated with low or lack of intention to publish in OAJ platform among researchers and institutions. This scenario has been observed in most of the higher learning institutions where dissertations and theses are on library shelves with little efforts to disseminate to the outside community (Francescon, 2017). In the same line, most of institutions lack policies on OAJ publishing as well as on repository, which inhibits individual's intention to publish in OAJ.

Based on the above findings, it can be deduced that despite the tremendous development of OAJ, most of the articles are not published on this platform. The literature available reveals a minimal utilization of OAJ for knowledge dissemination which appeals for more studies. In essence, there seems to be limited theoretical models which can significantly assess researchers' intention to publish in OAJ. In that regards, most researchers have exhibited low intention to publish in OAJ. Thus, this study fills the gap by exploring the factors which influence researchers' intention to publish in OAJ, since the intention to publish in OAJ also determines certain behaviour of individuals (Massoro \& Othman, 2016; Yaghoubi \& Bahmani, 2010). The dearth of research on the factors which influence intention to publish in OAJ is an indication that most scholars are yet to synchronize the importance of OAJ. Consequently, this study aims to identify the factors that influence researchers' intention to publish in open access journals. It also identifies the strongest predictors of citation impact towards intention to publish in open access journals.

\section{Theoretical Framework \\ 3.1. Journal Reputation}

The publication of open access journals has led to the proliferation of online publishers worldwide. The number of publishers as well as that of Open Access Journals has increased immensely over time (Directory of Open Access Journal, 2018). Similarly, the number of predatory journal publishers has been increasing at an alarming rate, thereby raising doubt about the quality and impact of OAJ platforms. Hence, a need for monitoring the quality of OAJ reputation like that of traditional journals is pivotal for its success and sustainability (Masten \& Ashcraft, 2016). As an authors or readers, it is highly necessary and encouraged to check journal reputation in order to ensure that the right information is sourced from authentic sources. This is in harmony with Beall's report which expressed the concern on the mushrooming of predatory journals which rose from 18 in 2011 to 923 in 2015 (Beall, 2016). Authors and readers are highly warned and reminded to beware of the deceptive publishers and predatory journals which have created a pervasive environment in OAJ publishing (Poltronieri, Bravo, Curti, Ferri, \& Mancini, 2016). Thus, authors are highly encouraged to cross-check the authenticity and reputation of journals before submitting their articles for publishing (Dallmeier-Tiessen et al., 2011).

On the other hand, writing of scholarly articles requires the citation of previous studies so as to justify or identify the gap in literature. Thus, authors usually cite the references which are published in reputable journals and with high impact factor. Consequently, journal reputation has a great contribution to the citation impact of the article which might motivate the researchers' intention to publish in OAJ. In other words, the reputation of journals is believed to influence an individual's intention to submit an article for publication. This is due to fact that a journal's reputation denotes how researchers perceive, position and rank that particular journal in terms of citation impact, journal impact factor, editorial board performance and peerreview quality. Hence, the following hypotheses were tested:

H1: Journal reputation has a significant effect on intention to publish in OAJ

H2: Journal reputation has a significant effect on research citation impact

\subsection{Speed of Publishing}

It is believed that an author's decision to select a particular journal depends on the extent he/she thinks publishing in open access journals is faster compared to the traditional subscription journals (Masrek \& Yaakub, 2015). This owes to the fact that journals which offer speed publishing bid additional criterion for its selection (Tennant et al., 2016) and gain a higher possibility of being one-stop centre for research publications. Researchers are mostly concerned with publishers who offer speed publishing in order to enhance article visibility, career advancement and citation impact (Bjork \& Holmstrom, 2006). Nariani and Fernandez (2012) study also revealed that author decision to select the OAJ rest on the speed of publishing which includes 
easiness, fastness, and turnaround. Thus, authors perceive the speed of publishing to enhance the article's visibility and wider readership which led to citation impact. Bjork and Holmstrom (2006) study on benchmarking scientific journals from the submitting author's viewpoint documented that the speed of publication is an important factor which directly influences citation impact as well as the intention to publish either in OAJ or subscription journal. As such, this study hypothesized that:

H3- Speed of publishing is a significant predictor of the citation impact of OAJ.

\subsection{Content Relevance}

Content relevance covers a wide range of the criteria for publishing in OAJ. Thus, content relevance denotes the extent to which the journal's scope fits with the scholar's subject interest. It mostly concerns how an individual values the contents and context covered by publisher in a particular OAJ in terms of its significance, coverage, currency, accuracy and authenticity (Nariani \& Fernandez, 2012; Nowick, 2008). In essence, content relevance reflects the extent to which a scholar believes that the subject content is consistent with his/her interest. In that case, the intention to publish in OAJ depends on how authors weigh the compatibility of the contents presented on previous journal issues. Tennant et al. (2016) and Park (2009) studies revealed that content compatibility also significantly affects scholars' intention to publish in open access, where Masrek and Yaakub (2015) documented that perceived topical compatibility is one of the main predictors of authors' intention to publish in OAJ. This is due to the fact that OAJ offers relevant contents in terms of coverage and they usually undergo blind peer review by subject experts (Suber, 2004). The relevant contents presented by OAJ also provide a possibility for wide citation impact of a particular article (Nariani \& Fernandez, 2012). Contrary to expectation, for decades, some of the authors have consistently believed that the OAJ bypass peer review process (Obuh \& Bozimo, 2012) which is detrimental to the quality of the contents. Even though the open access awareness campaigns have been on for years, the irony is that misconceptions about the quality of the OAJ in terms of content have persisted alongside its growth (Rodriguez, 2014). Scholars' mindsets on the OAJ have consistently devalued the contents as they are perceived to have little or no quality control measures which affect the citation impact in one way. Consistent with the above findings, it is therefore hypothesized that:

H4- Content relevance is a significant predictor of citation impact; and H5- Content relevance is a significant predictor of intention to publish in OAJ.

\subsection{Visible Advantage}

Visible advantage refers to the extent to which the audience has accessibility to the contents published in OAJ (Park, 2009). In other words, visible advantage reflects the extent to which a scholar believes that publishing in OAJ offers a high possibility of the article visibility. Through OAJ publishing outlet, the articles can be accessed by multiple readers and receive the frequent citation (Xia, Myers, \& Wilhoite, 2010). Davis (2011) and Gasparyan (2013) contended that articles published in OAJ achieve ubiquitous access regardless of an individual's is connection to the internet. Thus, the visible advantage offered by OAJ ensures a rapid dissemination of knowledge to a wider audience without a time limit. As such, an article published in OAJ has attains a citation impact which on the other hand influences the author's intention to publish. The study conducted by Mann, Walter, Hess, and Wigand (2009) among 481 researchers revealed that their intention to publish is significantly influenced by the capability to disseminate new findings widely and rapidly. Similarly, Tennant et al. (2016) observed that visible advantages also predict a wide audience which facilitates citation impact of the article. This is due to fact that readers mostly consult and refer to the articles which are accessible to them. That means a visible article is open to wider readership, which might possibly influence readers to make reference. It can be deduced that the visibility of an article has a significant contribution to wider readership and citation impact which in one way or another influences the researcher's intention to publish in OAJ. Based on the aforementioned, it is hypothesized that:

H6: Visible advantage has a significant effect on research citation impact

H7: Visible advantage has a significant effect on intention to publish in OAJ

\subsection{Citation Impact}

Citation impact is used to indicate an average number of citations received on a particular document. Citation impact is used as an indicator which plays a great role in research evaluation (Waltman, 2016). It has received extensive attention in the bibliometric and scientometric literature, and has been regarded as a vital indicator in the evaluation of research performance. Citation impact can be employed at all organizational levels such as authors, institution, country, region and discipline. The proliferation of OAJ publishing also is considered to provide a high possibility of a citation impact of an article. Xia et al. (2010) revealed that the articles published in open access receive more citation impact compared to non-open access. In the same vein, Koler-Povh, Južnič, and Turk (2014) compared the citation impact of open access and non-open access in the field of civil engineering. The results revealed that open access articles received $5 \%$ significant level. Salisbury, Chowdhury, and Smith (2017) argued that articles published in OAJ particularly in the Web of Sciences and Google Scholars from the year 2014 to 2015 display a high citation impact than non-open access. Thus, the 
articles which are published in OAJ receive a high citation impact as they are freely accessible to a wider audience at any time. The advantage of citation impact on OAJ has also been documented by Norris, Oppenheim, and Rowland (2008) where the articles under Toll access received a marginal citation impact compared to open access articles in the field of ecology, applied mathematics, sociology and economics. With toll access, the readers or institution should pay for one-time access fees or annual subscription fees which are cost-prohibitive to these institutions. Regarding the toll access, all institutions or readers without subscription fees are screened out, which denies accessibility and the possibility to make references. Therefore, OAJ supersedes all potential modes of scholarly communication through enabling free and unrestricted re-use of the research findings as well as provision of permanent availability of the article on the internet, thereby enhancing citation impact of the article and enabling knowledge sharing. Poltronieri et al. (2016) also observed that the impact factor of the OAJ has gradually risen with prevalence for medicine and biological sciences. In concord with the above findings, this study hypothesized that:

H8- Citation impact is a significant predictor of intention to publish in OAJ

\section{Conceptual Framework and Research Model}

The purpose of this study is to identify the factors influencing intention to publish in open access journals among researchers in Tanzania research institutes. Based on the theoretical framework as documented from previous studies, this study evaluates the influence of journal reputation, citation impact, and content relevance on intention to publish. Coupled with the empirical background on the factors influencing intention to publish in open access journals, the research model is presented below Figure 1.

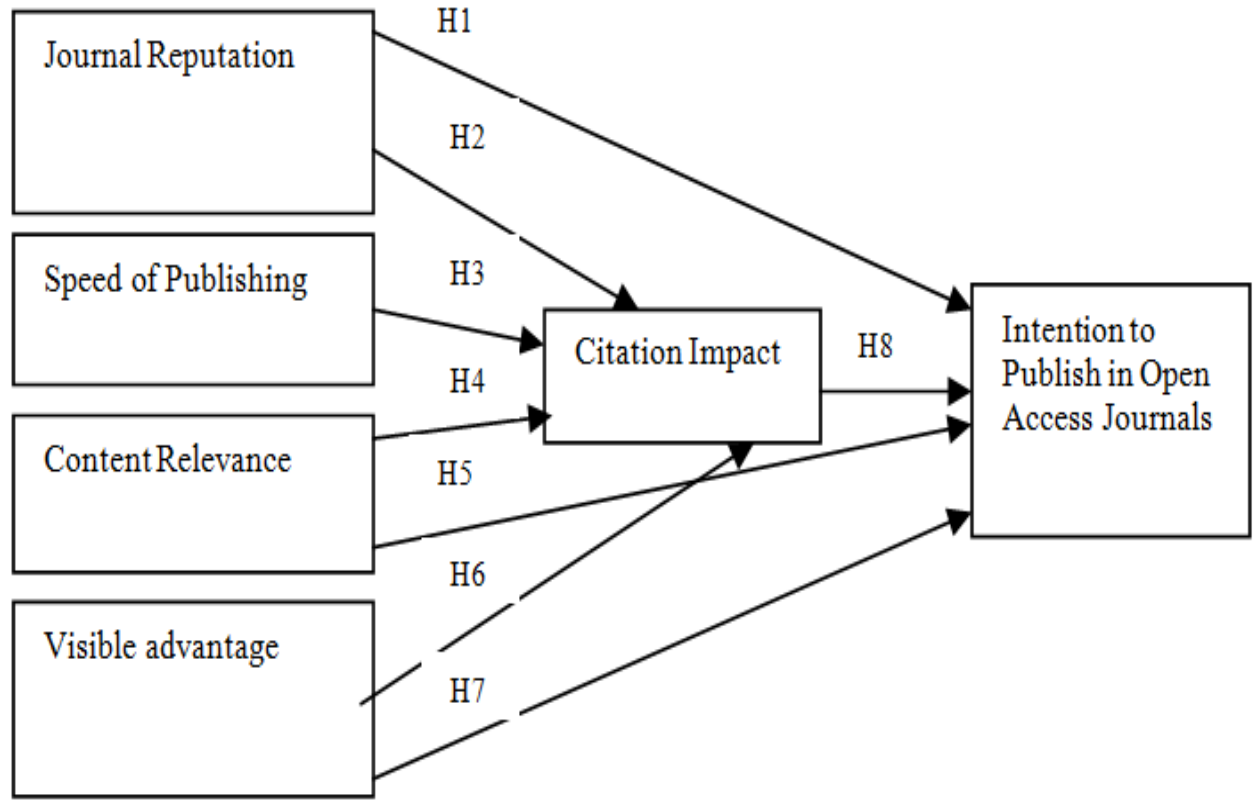

Figure-1. Research Model.

\section{Research Methodology}

This study adopted a survey method; hence, questionnaire was used to collect data. The questionnaire reflects six constructs adopted and adapted from the previous studies. The adapted questions were validated by subject experts in order to ensure coherence, reliability and consistency. The questionnaire items were pretested with several experts, including 60 researchers from Agricultural Research Institutes (ARIs).

The study was carried out in three agricultural zones which are: Eastern, Central and Southern zone. The choice of these agricultural zones is based on the fact that they are among the well established agricultural research institutes in Tanzania and they also have a large number of researchers dealing with the different fields of agriculture, livestock and fisheries. The study population consists of agricultural researchers working in the Ministry of Agriculture, Livestock and Fisheries (MALF) under the Directorate of Research Division (DRD) in Tanzania. A total of 149 questionnaires were sent to the targeted researchers in the selected ARIs. Data were collected for two months where a total of 138 (85.2\%) questionnaires were returned and only 121 $(81.2 \%)$ were used for further analysis.

\subsection{Data Analysis and Findings}

The Statistical Package for Social Science (SPSS) and Partial Least Square-Structural Equation Modeling (PLS-SEM) were used in analysis. The SPSS 23.0 version was used to carry out descriptive analysis of the demographic characteristics of the respondents. Further, PLS-SEM Smart PLS 2.0 M3 was used to assess the 
measurement model, the structural model and for hypotheses testing. The choice of PLS path modeling was based on the ground that it is one of the statistical methods for structural equation modeling which follows path-analytics modeling procedures (Reinartz, Haenlein, \& Henseler, 2009). The PLS path modeling (SEM) provides solid results even in a complex model, small sample and it is flexible (Hair, Ringle, \& Sarstedt, 2013); it also evaluates both formative and reflective measurement model (Henseler, Ringle, \& Sarstedt, 2015). Thus, the PLS-SEM path modeling analysis involves factor analysis, evaluation of validity and reliability of the measurement instruments. In the assessment of the structural model, the coefficient of determinant, path coefficient and effect sizes were used, while the path coefficient and t-statistics were used for hypotheses testing.

\subsection{Demographic Profile of Respondents}

The demographic profile of respondents was analyzed in terms of gender, age, education level and designation. A total of 121 respondents comprising $85(70.2 \%)$ males and $36(29.8 \%)$ female were contacted. Majority of respondents 52 (43\%) were between ages 36-45 years, followed by the age group 26-35 years which comprises $35(28.9 \%)$ respondents. In all age groups, the number of males was more compared to females. A large number of males in agricultural research have been attributed to the education specialization especially science subject. Table 1 showcases the demographic profile of the respondents.

Table-1. Demographic Profile of the Respondents.

\begin{tabular}{l|l|l|c}
\multicolumn{5}{c}{ Table-1. Demographic Profile of the Respondents. } \\
\hline & No. of Items & Frequency & Percent \\
\hline Gender & Male & 85 & $70.2 \%$ \\
\hline Total & Female & 36 & $29.8 \%$ \\
\hline Position & $\begin{array}{l}\text { Principal Agriculture Research } \\
\text { Officer }\end{array}$ & $\mathbf{1 2 1}$ & $\mathbf{1 0 0 . 0}$ \\
\hline & Senior Agriculture Research Officer & 15 & $23.1 \%$ \\
\hline & Research Officer & 53 & $12.4 \%$ \\
\hline & Assistant Research Officer & 25 & $43.8 \%$ \\
\hline Total & & 121 & $20.7 \%$ \\
\hline Age Group & $26-35$ & 35 & 100 \\
\hline & $36-45$ & 52 & $28.9 \%$ \\
\hline & $46-55$ & 19 & $43 \%$ \\
\hline & $>55$ & 15 & $15.7 \%$ \\
\hline Total & & $\mathbf{1 2 1}$ & $12.4 \%$ \\
\hline Source: Field Survey, Dec, 2015- Jan, 2016. & & $\mathbf{1 0 0 . 0}$
\end{tabular}

Referring to statistics from the Tanzania Ministry of Education between 1998 and 2008, it was observed that more male students enrolled in science subject compared to the female students (The United Republic of Tanzania, 2016) consequently, this has an effect on career specification. Hence, this could be a likely reason for the larger number of males in the Agricultural Research Institutes than their female counterparts. In terms of work positions of the respondents, 28 (23.1\%) were Principal Agricultural Research Officers (PARO), 15 (12.4\%) Senior Agricultural Research Officers (SARO), 53 (43.8\%) Researchers and 25 (20.7\%) Assistant Researchers. The study also found that only $24(19.8 \%)$ of the respondents have published their research findings in open access journals while 97 (80.2\%) had never published.

\subsection{Assessment of the Measurement Model}

PLS-SEM was used for the assessment of the measurement model which includes the analysis of indicator outer loadings (factors analysis), reliability, internal consistency reliability, convergent validity and discriminant validity. The results indicate that all factors load within the recommended value, ranging from 0.670 to 0.938 . The results of Cronbach's alpha ranged from 0.702 (visible advantage) to 0.823 (content relevance) which is above the recommended threshold of 0.7 (Hair, Ringle, \& Sarstedt, 2011). The Composite Reliability $(\mathrm{CR})$ was used for the assessment of internal consistency. The $\mathrm{CR}$ values of the constructs were above the recommended threshold of 0.5. The $\mathrm{CR}$ values ranged from 0.829 to 0.892. In addition, the Average Variance Extracted (AVE) result was above the recommended threshold of 0.5 (Henseler, Ringle, \& Sinkovics, 2009) ranging from 0.585 to 0.777 . Thus, the results obtained indicate that the measurement model meet the assessment criteria. Table 2 presents the results of Cronbach's alpha, composite reliability and R-Square of the constructs. 
Table-2. Cronbach's Alpha, Composite Reliability and R-Square.

\begin{tabular}{l|l|l|l}
\hline Variables & Cronbach's Alpha & Composite Reliability & R-Square \\
\hline Citation Impact & 0.708743 & 0.837256 & 0.318860 \\
\hline Content Relevance & 0.823073 & 0.892229 & \\
\hline Intention to Publish & 0.768570 & 0.849164 & 0.404202 \\
\hline Journal Reputation & 0.803662 & 0.884345 & \\
\hline Speed of Publishing & 0.716770 & 0.874077 & \\
\hline Visible Advantage & 0.701627 & 0.829449 & \\
\hline \multicolumn{2}{l}{ Source: Field Survey, Dec, 2015- Jan, 2016.}
\end{tabular}

The convergent validity assesses the extent to which a measure correlates positively with an alternative measure of the same constructs (Hairs, Hult, Ringle, \& Sarstedt, 2016). The results also show that the constructs are highly correlated among themselves, and the AVE values for all constructs are greater than the squared correlation with other constructs, at the same time, has less correlation with measures of distinct constructs. The convergent validity values for the constructs ranged from 0.691 to 0.837 . The AVE values exceeded the recommended threshold of 0.5 , demonstrating adequate convergent validity.

The study also assessed the discriminant validity in order to measure the extent to which a construct is truly distinct from other constructs by empirical standards. Referring to Campbell and Fiske (1959) the discriminant validity assesses the extent to which a construct is unique and how it captures phenomenon not presented by another construct in the model. The rule of the thumb suggests that loadings should be at least 0.32, where the loadings from 0.45 to 0.54 are considered fair, 0.55 to 0.62 are considered good, 0.63 to 0.70 are considered very good and above 0.71 are considered excellent (Hairs et al., 2016). Thus, the cross-loadings result of the indicators under the study loaded higher on their own constructs, which indicates satisfactory discriminant validity (Hair et al., 2011). Apparently, the variables under study are discriminant to each other as they load on their respective constructs. Similarly, the square root of the constructs' AVE shows values that exceed the correlations between the constructs which falls within the recommended threshold 0.71 and is considered as excellent (Henseler et al., 2009).

Table-3. Convergent Validity and Discriminant Validity.

\begin{tabular}{l|l|l}
\hline Constructs & AVE & $\sqrt{\text { AVE }}$ \\
\hline Citation Impact & 0.632 & 0.795 \\
\hline Content Relevance & 0.735 & 0.857 \\
\hline Intention & 0.585 & 0.765 \\
\hline Journal Reputation & 0.718 & 0.847 \\
\hline Speed of Publishing & 0.777 & 0.881 \\
\hline Visible Advantage & 0.622 & 0.789
\end{tabular}

The results obtained from the assessment of measurement model demonstrate that the model is reliable and valid; thus, the need to proceed with the assessment of the structural model.

\subsection{Assessment of the Structural Model}

The assessment of the structural model was performed in order to evaluate its validity and to test the hypotheses under study. The assessment of the structural model involves the tests of the coefficient of determination $\left(\mathrm{R}^{2}\right)$; path coefficient; effect size $\left(\mathrm{f}^{2}\right)$; and predictive relevance $\left(\mathrm{Q}^{2}\right)$. The coefficient of determination $\left(\mathrm{R}^{2}\right)$ value is commonly used in the assessment of structural model in order to measure the model predictive accuracy. The calculation of R-Square and the path coefficients were performed in order to determine the effect of exogenous latent variables on the endogenous variables; with reference to the rule of thumb of 0.01, 0.09, and 0.25 indicating small, medium and large exploratory power respectively (Hairs et al., 2016; Mitchell \& Jolley, 2013). The result of R-square is 0.404 for intention to publish and 0.319 for citation impact. The result of the $\mathrm{R}^{2}$ falls within the recommended threshold of above 0.01, demonstrating strong explanatory power Figure 2. This means that journal reputation, citation impact, content relevance and visible advantage contribute $40.4 \%$ to intention to publish, and the remaining $59.6 \%$ accounts for other factors that are not discussed in this study. In addition, journal reputation, speed of publishing, content relevance as well as visible advantage account for $31.9 \%$ of the citation impact, while $69.1 \%$ is explained by other factors that are not included in this study. 


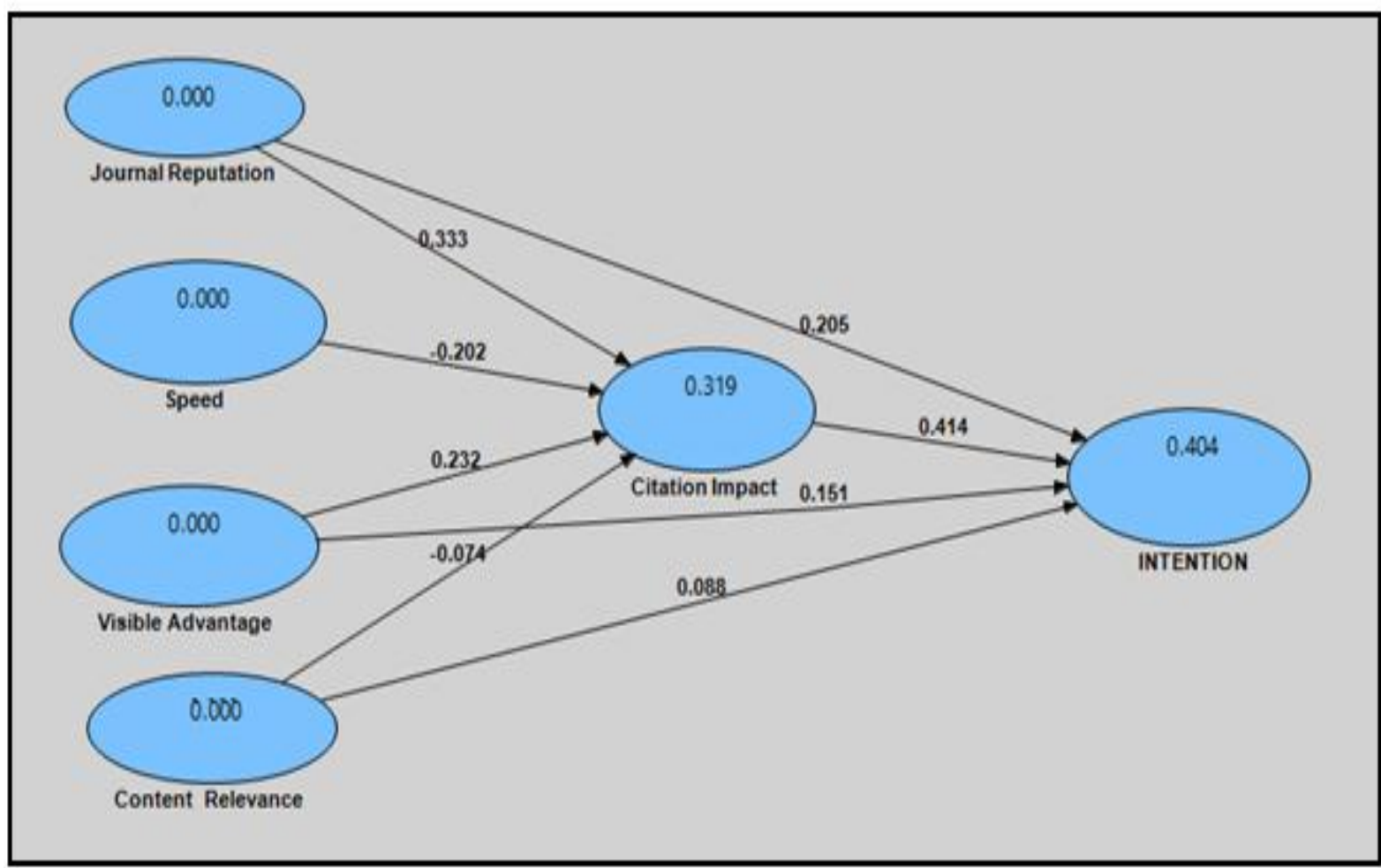

Source: PLS Output.

Figure-2. Coefficient of Determination $\left(\mathrm{R}^{2}\right)$.

Further, the predictive relevance $\left(\mathrm{Q}^{2}\right)$ value of the dependent variables was performed through blindfolding procedure. The $Q^{2}$ measures the observed values reconstructed by the model and its parameter estimates should be higher than zero (Henseler et al., 2009). Thus, the $\mathrm{Q}^{2}$ results were above the recommended value of zero which indicating an adequate predictive relevance of the model as demonstrated in Table 4.

Table-4. Coefficient of Determination and Predictive Relevance.

\begin{tabular}{|c|c|c|}
\hline Constructs & $\mathbf{R}^{2}$ & $\mathbf{Q}^{2}$ \\
\hline Citation Impact & 0.319 & 0.200 \\
\hline Intention to Publish & 0.404 & 0.223 \\
\hline
\end{tabular}

In addition, the effect size values obtained were within the recommended threshold of $0.02,0.15$, and 0.35 , indicating small, medium and large effect respectively (Henseler et al., 2009). The findings indicate that both visible advantage (0.446) and content relevance (0.604) have large effect on intention to publish, followed by journal reputation (0.331). Furthermore, speed and visible advantage have medium effect on citation impact as demonstrated in Table 5.

\begin{tabular}{l|l|l}
\multicolumn{4}{c}{ Table-5. Effect Size. } \\
\hline Constructs & Effect Size & Remarks \\
\hline Citation Impact -> Intention & 0.122 & Small \\
\hline Content Relevance -> Citation Impact & 0.134 & Small \\
\hline Content Relevance -> Intention & 0.604 & Large \\
\hline Journal Reputation -> Citation Impact & 0.144 & Small \\
\hline Journal Reputation -> Intention & 0.331 & Medium \\
\hline Speed -> Citation Impact & 0.201 & Medium \\
\hline Visible Advantage -> Citation Impact & 0.315 & Medium \\
\hline Visible Advantage -> Intention & 0.446 & Large
\end{tabular}

\subsection{Hypothesis Testing}

Hypothesis testing was performed through PLS algorithm and bootstrapping test. PLS algorithm was performed by looking at the path coefficient while the bootstrapping test was performed by drawing a large number of subsamples from the original data with replacement and estimates models for each subsample. The bootstrapping was performed in order to determine standard errors of coefficient estimates and to assess the coefficient statistics (t-test) significance without relying on distribution. The PLS algorithm and 
bootstrapping test results are normally used to make a decisions either to support or refute the hypothesis as displayed in Table 6.

Table-6. Hypotheses Testing.

\begin{tabular}{l|l|l|l|l}
\hline Hypothesis & Relationship & Path Coefficient & T-Value & Conclusion \\
\hline H1 & Journal Reputation -> Intention & 0.2053 & $3.062^{* *}$ & Supported \\
\hline H2 & Journal Reputation -> Citation Impact & 0.3331 & $3.959^{* *}$ & Supported \\
\hline H3 & Speed -> Citation Impact & -0.2018 & $2.512^{*}$ & Not Supported \\
\hline H4 & Content Relevance ->Citation Impact & -0.0736 & 1.280 & Not Supported \\
\hline H5 & Content Relevance -> Intention & 0.0880 & 1.614 & Not Supported \\
\hline H6 & Visible Advantage -> Citation Impact & 0.220811 & $4.031^{* *}$ & Supported \\
\hline H7 & Visible Advantage -> Intention & 0.150965 & $2.757^{*}$ & Supported \\
\hline H8 & Citation Impact -> Intention & 0.414574 & $6.244^{*}$ & Supported \\
\hline Note: * Significance at t value $\geq 1.96$ with p $\leq 0.05, * *$ Significance at t value $\geq 2.59$ with $\mathrm{p} \leq 0.01$. & &
\end{tabular}

Based on the findings presented in Table 6, it can be inferred that journal reputation has a significant influence on intention to publish $\left(\mathrm{H}_{1}: \beta=0.2, \mathrm{t}=3.06\right)$; thus, $\mathrm{H}_{1}$ is supported. Also, journal reputation has a significant effect on citation impact $\left(\mathrm{H}_{2}: \beta=0.3, \mathrm{t}=3.95\right)$. Although the path coefficient for the relationship between speed and citation impact was greater than 0.1 and has a t-statistic value greater than $1.96(\beta=-0.2$, $\mathrm{t}=2.51)$, the third hypothesis was not supported because the negative sign found on the path coefficient indicates that there is an inverse relationship between speed and citation impact, which is contrary to the study proposition based on past existing literature. In such situation, Urbach and Ahlemann (2010) opined that such hypothesis should be rejected. Hypothesis four which assessed the influence of content relevance on citation impact showed that there is an insignificant relationship between both constructs $\left(\mathrm{H}_{4}: B=-0.0736\right.$, $\mathrm{t}=1.28)$. Thus $\mathrm{H}_{4}$ was not supported. In the same vein, hypothesis five $(\mathrm{H} 5)$ was not supported as content relevance was found to have no significant effect on intention to publish in OAJ $(\beta=0.08, t=1.61)$. Meanwhile, visibility advantage has a positive significant effect on citation impact $\left(\mathrm{H}_{6}: B=0.22, t=4.03\right)$ and Visible advantage has a significant influence on intention $\left(\mathrm{H}_{7}: \beta=0.15, \mathrm{t}=2.75\right)$. Lastly, the result shows that citation impact has a positive significant effect on intention to publish. Thus, H8 was also supported $(\beta=0.4145, t=$ 6.244). In summary, five of the eight hypotheses under study were supported while the other three were not.

\section{Discussion}

This study assessed the factors that may influence direct and indirect intention to publish in open access journals among researchers in Tanzania agricultural research institutes. Five independent variables (journals reputation, visible advantage, and content relevance, speed of publishing and citation impact) were used to predict the intention to publish in open access journals. The results showed that three variables (journal reputation, visible advantage, and citation impact) have the significant effect on intention to publish while the other two (speed of publishing and content relevance) have no significant effect. The results revealed that journal reputation has a significant effect on intention to publish in OAJ, which is consistent with the findings of Masrek and Yaakub (2015) and Nariani and Fernandez (2012) as these studies revealed that journal reputation has a significant influence on intention to publish and the selection of a specific OAJ. How ever, this finding contradicts that of Ayeni and Adetoro (2017) which found that the intrusion of predatory publishers on OAJ has detriment on its reputation. The findings also demonstrated that journal reputation has a significant positive effect on citation impact (H2: path coefficient: $\beta=0.3331, \mathrm{t}$-value: 3.959). However, none of these previous studies has assessed the influence of journal reputation on citation impact. It is therefore noteworthy to mention that this study contributes to the creation of a theoretical link between journal reputation and citation impact as well as intention to publish. The result suggests that the higher the journal reputation, the higher would be the citation impact of the OAJ.

The assessment of the influence of speed of publishing on citation impact revealed that the hypothesis was not supported. This result indicates that the speed of publishing the articles is not a significant predictor of the citation impact of an article published in OAJ, which contradicts expectation. Hence, the speed of publishing does not guarantee citation impact; rather, other factors which were not considered in this study are responsible for enhancing the citation impact. Content relevance was also found to have an insignificant effect on citation impact and the intention to publish in OAJ. It is believed that most authors access the content of journals for making valuable judgments in terms of topic relevance, compatibility and coverage. This result is in concord with Park (2009) study which revealed that perceived topic compatibility has insignificant effect on the decision to publish in a web-based scholarly publishing. Further, findings show that visible advantage has a significant effect on citation impact as well as intention to publish in open access. Similarly, the findings indicate that the higher the citation impact, the higher the intention to publish in OAJ. The effect of visible advantage on citation impact is significant and the same was observed for its effect on the intention to publish in OAJ. This is due to fact that most researchers believe that publishing in OAJ provides the high possibility of 
the article to be seen by the large audience and downloaded widely. This result is in tandem with Masrek and Yaakub (2015) study which revealed that visible advantage facilitates a wider readership which motivates scholars to publish in OAJ. Nonetheless, this finding suggests that as visible advantage increases among the researchers, the possibility for the researchers to publish in OAJ will sparingly increase. This result is consistent with prior research findings by Salisbury et al. (2017) on the scholarly impact of open access (OA) versus Non-OA on the articles published in Web of Sciences and Google Scholars from the year 2014 to 2015. Salisbury et al. (2017) reported that open access articles receive higher citation than Non-Open Access articles. The present results concur with Koler-Povh et al. (2014) and Nariani and Fernandez (2012) that OAJ receives more citation impact compared to non-OAJ. This finding supports Musa (2016) and Xia et al. (2010) study which documented that multiple access availability of open access articles has a positive impact on citation impact. It was postulated that authors do strive for a good chance of citation in order to gain promotion and to prosper in their career (Waltman, 2016). Intention to publish in OAJ achieved a high R-square which revealed that the constructs under study have high prediction rate towards intention. This result is consistent with that of Massoro and Othman (2017) study which showed that intention is predicted by a number of factors. Generally, the intention stands as a motivation for the intention to publish in OAJ.

\section{Conclusion}

This study assessed the factors that influence the intention of researchers in Agricultural Research Institutes (ARI) in Tanzania to publish in open access journals. To achieve this, an empirically based framework mainly drawn from the literature review was developed. The results of the analyses suggest that journal reputation, visible advantage and citation impact have significant effects on the intention to publish in open access journals. Similarly, the results reflect that journal reputation and visible advantage are the truly significant predictors of citation impact of an article. Thus, the researchers normally publish the research article in reputable journals with a high expectation of being cited; which is important for career advancement and institution reputation. The results reveal that journal reputation has a significant effect on attracting a wider audience, which is the prime concern in winning a chance for citation impact. However, the speed of publishing and content relevance indicates negative effect on citation impact. Thus, the availability of online submission and immediate peer reviewing process which enhances fast publication once the article is accepted compared to traditional journals is not a predictor of citation impact. This study provides both theoretical and practical contributions to the understanding of the determinants of citation impact as well as intention to publish in an open access journal.

\section{Recommendation}

The importance of OAJ in terms of its permanent availability and the possibility of reaching a wide audience are fundamental for enhancing the reputation of researchers and institutions at large. The importance of publishing in OAJ should be more emphasized in research institutions than other organizations since knowledge creation, knowledge exchange, transfer and utilization are the core of their work. Therefore, it is important for these institutions to develop and harness the appropriate environment that facilitates publishing in OAJ, in order to enhance knowledge sharing and creation of a knowledge-based society. Management staff should guide researchers through highlighting the reputable journals where researchers can communicate their research findings.

\section{Implications}

The research findings reveal that the intention to publish in OAJ is determined by the journal's reputation, citation impact, and visible advantage. However, the speed of publishing has an insignificant impact on intention to publish. Therefore, the effect of journal reputation in term of the reputation of the peer reviewers, editors and adherence to international standards are vital in raising the researchers' intention to publish rather than the speed of publishing. The findings are expected to help decision and policy makers in research institutes in Tanzania and other African countries, since the research institutes are considered as the factory for knowledge-creation and dissemination.

\section{References}

Ayeni, P. O., \& Adetoro, N. (2017). Growth of predatory journals: Implication for quality assurance in library and information science research. Library Hi Tech News, 34(1), 17-22.

Beall, J. (2016). Beall's list of predatory publishers 2015. Retrieved from https://scholarlyoa.com/.

Berlin Declaration on OA Knowledge. (2003). Berlin declaration on open access to knowledge in the sciences and humanities (22 Oct, 2003). Retrieved from www. zim.mpg.de/openaccessberlin/berlin_declaration.pdf. [Accessed, on 23rd April 2018].

Bjork, B. C., \& Holmstrom, J. (2006). Benchmarking scientific journals from the submitting author's viewpoint. Learning Publishing, 19(2), 147-155.

Brown, O. P., Cabell, D., Chakravarti, A., Cohen, B., Delamothe, T., Michael, E., \& Les, G. E. M. (2003). Bethesda statement on open access publishing. Journal of Library and Information Science, 13(2), 1-7. 
Budapest Open Access Initiative. (2002). Budapest open access initiative. (14 Feb, 2002). Retrieved from http://www.soros.org/openaccess/read.shtml. [Accessed, 23rd April, 2018].

Campbell, D. T., \& Fiske, D. W. (1959). Convergent and discriminant validation by the multitrait-multimethod matrixi. Psychological Bulletin, 56(2), 81-105.

Dallmeier-Tiessen, S., Darby, R., Goerner, B., Hyppoelae, J., Igo-Kemenes, P., Kahn, D., \& Stelt, W. V. D. (2011). Open access journals - What publishers offer, what researchers want. Information Services and Use, 31(1), 85-91.

Davis, P. M. (2011). The impact of free access to the scientific literature: A review of recent research. Journal of Medical Library Association, 99(3), 208-217.

Directory of Open Access Journal. (2016). A list of journals added to DOAJ from 19th March 2014. Retrieved from https://doaj.org/.

Francescon, D. (2017). Research without borders: Sharing expertise in Africa. Retrieved from https://www.elsevier.com/ [Accessed on 23 April 2018].

Gasparyan, A. Y. (2013). Choosing the target journal: Do authors need a comprehensive approach? Journal of Korean Medical Science, 28(8), 1117-1119.

Hair, J. F., Ringle, C. M., \& Sarstedt, M. (2011). PLS-SEM: Indeed a silver bullet. The Journal of Marketing cognition and Practice, 19(20), 139-152.

Hair, J. F., Ringle, C. M., \& Sarstedt, M. (2013). Editorial: Partial least squares structural equation modeling: Rigorous applications, better results and higher acceptance. Long Range Planning, 46(1-2), 1-12.

Hairs, F. J., Hult, M. T. G., Ringle, M. C., \& Sarstedt, M. (2016). A primier on partial least squares structural equation modeling (PLS-SEM). Los Angeles: Sage.

Henseler, J., Ringle, C. M., \& Sinkovics, R. (2009). The use of partial least squares path modeling in international marketing. Advances in International Marketing, 20(1), 277-320.

Henseler, J., Ringle, M. C., \& Sarstedt, M. (2015). A new criterion for assessing discriminant validity in variance-based structural equation modeling. Journal of the Academy of Marketing Science, 43(1), 115-135.

Joshi, N., Vatnal, M., \& Manjunath, A. (2012). Open access initiatives: A boon to academic libraries. Library Philosophy and Practice (E-Journal). Paper No. 792.

Koler-Povh, T., Južnič, P., \& Turk, G. (2014). Impact of open access on citation of scholarly publications in the field of civil engineering. Journal of Scientometrics, 98(2), 1033-1045.

Mann, F., Walter, B. V., Hess, T., \& Wigand, R. T. (2009). Open access publishing in science. Communications of the ACM, 52(3), 135-138.

Masrek, M. N., \& Yaakub, M. S. (2015). Intention to publish in open access journal: The case of multimedia university Malaysia. Procedia - Social and Behavioral Sciences, 174(1), 3420 - 3427. Available at: 10.1016/j.sbspro.2015.01.1013.

Massoro, Z. Z., \& Othman, R. (2016). Determinants for usage of open access journals: An exploratory analysis. Paper presented at the e-Proceeding of the Global Conference of Information Technology and Multimedia (IT-M2016).

Massoro, Z. Z., \& Othman, R. (2017). Antecedents for actual usage intentions of open access journals in agricultural research institutions in Tanzania. Library Philosophy and Practice (E-Journal), Paper No. 1460: 1-19.

Masten, Y. B., \& Ashcraft, A. S. (2016). The dark side of dissemination: Traditional and open access versus predatory journals. Nursing Education Perspectives, 37(5), 275-277.

Miguel, S., Tannuri, D. O. E. F., \& Grácio, M. C. (2016). Scientific production on open access: A worldwide bibliometric analysis in the academic and scientific context. Publications, 4(1), 1-15.

Mitchell, M. L., \& Jolley, M. J. (2013). Research design explained (8th ed.). CA, USA: Wadsworth Cengage Learning.

Musa, A. U. (2016). An appraisal of open access utilization among academic librarians in Federal Universities in Northwestern States of Nigeria. Library Philosophy and Practice (E-Journal). Paper No. 1374.

Nariani, R., \& Fernandez, L. (2012). Open access publishing: What authors want. College \& Research Libraries, 73(2), 182195.

Norris, M., Oppenheim, C., \& Rowland, F. (2008). The citation advantage of open-access articles. Journal of the American Society for Information Science and Technology, 59(12), 1963-1972.

Nowick, E. A. (2008). Academic rank of authors publishing in open access journals. Agricultural Information Worldwide, 1(2), 45-51.

Nwagwu, E. W. (2013). Open access initiatives in African-structure incentives and disincentives. The Journals of Academic Librarianship, 39(1), 3-10.

Obuh, A. O., \& Bozimo, D. O. (2012). Awareness and use of open access scholarly publications by LIS lecturers in Southern Nigeria. International Journal of Library Science, 1(4), 54-60.

Park, J. H. (2009). Motivations for web-based scholarly publishing: Do scientists recognize open availability as an advantage? Journal of Scholarly Publishing, 40(4), 343-369.

Poltronieri, E., Bravo, E., Curti, M., Ferri, M., \& Mancini, C. (2016). Open access publishing trend analysis: Statistics beyond the perception. Information Research, 21(3), 1-13.

Reinartz, W. J., Haenlein, M., \& Henseler, J. (2009). An empirical comparison of the efficacy of covariance-based and variance-based SEM. International Journal of Research in Marketing, 26(4), 332-344.

Rodriguez, J. (2014). Awareness and attitudes about open access publishing: A glance at generational differences. The Journal of Academic Librarianship, 40(6), 604-610.

Salisbury, L., Chowdhury, A., \& Smith, J. J. (2017). Faculty publications from a research university: The scholarly impact of open access versus non-open access. Journal of Science and Technology Libraries, 36(2), 187-199.

Suber, P. (2004). A very brief introduction to open access. [Accessed, 17th November 2014].

Tennant, J. P., Walder, F., Jacques, D. C., Masuzzo, P., Collister, L. B., \& Hartgerink, C. H. J. (2016). The academic, economic, and societal impacts of open access: An evidence-based review [version 3]. F1000 Research, 5, 632. Available at: 10.12688/f1000research.8460.3. 
The United Republic of Tanzania. (2016). Presidents office regional administration and local government: Pre-primary, primary and secondary education statistics in brief. Dodoma, Tanzania: Iyombe, Musa.

Urbach, N., \& Ahlemann, F. (2010). Structural equation modeling in information system research using partial least squares. Journal of Information Technology and Application, 11(2), 5-40.

Waltman, L. (2016). A review of the literature on citation impact indicators. Journal of Informetrics, 10(2), 365-392.

Ware, M., \& Mabe, M. (2015). The STM report: An overview of scientific and scholarly journal publishing, celebrating the 350th of journal publishing (4th ed.). The Hague: Prinns Willem Allexanderh: International Association of Scientific, Technology and Medical Publisher.

Xia, J., Myers, R. L., \& Wilhoite, S. K. (2010). Multiple open access availability and citation impact. Journal of Information Science, 37(1), 19-28.

Yaghoubi, N., \& Bahmani, E. (2010). Factors affecting the adoption of online banking: An integration of technology acceptance model and theory of planned behavior. International Journal of Business and Management, 5(9), 159-165. 